\title{
Comment on "Comparative Population Pharmacokinetics of Darunavir in SARS-CoV-2 Patients vs. HIV Patients: The Role of Interleukin-6"
}

\author{
Dario Cattaneo ${ }^{1,2}$ (C) Mario Corbellino ${ }^{3} \cdot$ Valeria Cozzi $^{1} \cdot$ Marta Fusi $^{1} \cdot$ Cristina Gervasoni ${ }^{2,3}$ (])
}

Accepted: 28 January 2021 / Published online: 17 April 2021

(c) The Author(s), under exclusive licence to Springer Nature Switzerland AG part of Springer Nature 2021

We read with interest the manuscript by Cojutti et al. [1], which documented that darunavir clearance and drug trough concentrations were, respectively, $60 \%$ lower and five-fold higher in patients with severe acute respiratory syndrome coronavirus 2 (SARS-CoV-2) compared with patients with human immunodeficiency virus (HIV) for the same daily drug dose ( $800 \mathrm{mg}$ once daily). These results are in agreement with previous findings showing four-fold higher lopinavir trough concentrations in patients with SARS-CoV-2 vs patients with HIV [2-4]. As both HIV protease inhibitors are substrates of cytochrome P450 3A4, the authors hypothesized that the reduced clearance of darunavir and lopinavir in patients with SARS-CoV-2 could have been driven by an impairment of drug metabolism related to the viral infection. Remarkably, interleukin-6 was the only clinical covariate that significantly correlated with darunavir pharmacokinetics. Accordingly, it has been proposed that the acute viral infection perpetrated a pro-inflammatory state that may, in turn, downregulate cytochrome P450 3A4 expression and activity, ultimately resulting in increased darunavir systemic concentrations $[1,5]$. As indirect support for this hypothesis, the authors have shown that lower interleukin-6 levels measured in the comparator group of patients with HIV were

This comment refers to the article available at https://doi. org/10.1007/s40262-020-00933-8.

An author's reply to this comment is available at doi:https://doi. org/10.1007/s40262-021-00996-1.

Dario Cattaneo

dario.cattaneo@asst-fbf-sacco.it

1 Unit of Clinical Pharmacology, ASST Fatebenefratelli Sacco University Hospital, Via GB Grassi 74, 20157 Milan, Italy

2 Gestione Ambulatoriale Politerapie (GAP) Outpatient Clinic, ASST Fatebenefratelli Sacco University Hospital, Milan, Italy

3 3rd Division of Infectious Diseases, ASST Fatebenefratelli Sacco University Hospital, Milan, Italy associated with higher darunavir clearance compared with patients with SARS-CoV-2.

Taken together, findings from lopinavir and darunavir can be considered as a proof of concept of viral disease-drug interactions, recalling the issue of optimal dose selection of the cytochrome P450 3A4 substrate in patients with SARSCoV-2. We believe, however, that the study by Cojutti et al. lacks a third mandatory comparator group: the patients co-infected with HIV and SARS-CoV-2. The inclusion of this additional group could allow the characterization of the pharmacokinetics of darunavir in patients with the concomitant presence of chronic and acute inflammatory states related to chronic and acute viral infections, respectively.

To address this issue, we searched through the database of our Clinic of Infectious Diseases for HIV-infected patients with a diagnosis of SARS-CoV-2 infection, given darunavir as maintenance antiretroviral therapy who underwent therapeutic drug monitoring of darunavir trough concentrations during COVID-19. Data on interleukin-6 and/or C-reactive protein were also collected as biomarkers of acute inflammatory progression in patients with SARS-CoV-2. To indirectly verify the comparability of the darunavir trough concentrations in patients with SARS-CoV-2 described by Cojutti et al., with those measured in our patients coinfected with HIV/SARS-CoV-2, we also considered a control group of patients with HIV not infected with SARS-CoV-2 undergoing therapeutic drug monitoring of darunavir trough concentrations (as done by Cojutti et al. [1]). Data were expressed as median (interquartile range); significance was set at $p<0.05$.

Six patients with HIV co-infected with SARS-CoV-2 and fulfilling the inclusion criteria were retrieved from the database of our clinic. They were mostly male (four out of six), with a median age of 62 (58-64) years. As shown in Table 1, no significant differences were found when comparing darunavir trough concentrations in patients co-infected with HIV/SARS-CoV-2 with patients mono-infected with HIV (836 [409-1523] vs 1273 [734-1954] ng/mL; $p=0.861$ ). 
Table 1 Comparison of darunavir trough concentrations and inflammatory indices in patients with SARS-CoV-2 and/or HIV infection from our cohort vs the Cojutti et al. cohort [1]

\begin{tabular}{lllll}
\hline & SARS-CoV-2 and HIV & HIV & SARS-CoV-2 & $\begin{array}{l}\text { HIV } \\
\text { Cojutti et al. [1] }\end{array}$ \\
\hline Patients, $n$ & 6 & 130 & 30 & 25 \\
Darunavir trough, ng/mL & $836(409-1523)$ & $1273(734-1954)$ & $4960(2015-7951)$ & $1010(550-2112)$ \\
Interleukin-6, pg/mL & $17(4-53)$ & n.a. & $31(10-115)$ & $2(2-3)$ \\
C-reactive protein, $\mathrm{mg} / \mathrm{L}$ & $21(10-35)$ & n.a. & n.a. & n.a. \\
\hline
\end{tabular}

Data were given as median (interquartile range)

$H I V$ human immunodeficiency virus, n.a. not available, SARS-CoV-2 severe acute respiratory syndrome coronavirus 2

We could not make formal statistical comparisons between our datasets and the those from Cojutti et al. (individual darunavir concentrations for the latest study were not available). However, to a visual evaluation, the darunavir trough concentrations measured in patients co-infected with HIV/ SARS-CoV-2 and patients mono-infected with HIV from our cohort fully matched to those measured in the comparator group of patients with HIV from the other study (1010 [550-2112] ng/mL), resulting in five-fold lower than darunavir trough concentrations measured in patients monoinfected with SARS-CoV-2 (4960 [2015-7951] ng/mL). No major differences in the inflammatory states were observed between the two cohorts of patients with SARS-CoV-2 (Table 1). Taken together, these findings provide indirect evidence that the observed differences in the darunavir trough concentrations between patients mono-infected with SARSCoV-2 and patients co-infected with HIV/SARS-CoV-2 were not biased by potential analytical issues (the concentrations of darunavir in patients mono-infected with HIV measured in the two studies were comparable) or affected by key differences in the inflammatory status between the two cohorts (patients co-infected with HIV/SARS-CoV-2 and patients mono-infected with SARS-CoV-2 had comparable interleukin-6 levels). Moreover, the darunavir trough concentrations measured in patients mono-infected with HIV and patients co-infected with HIV/SARS-CoV-2 were similar to those measured previously in healthy volunteers given darunavir at $800 \mathrm{mg}$ at steady state $[6,7]$. Hence, combined evidence from our study and the Cojutti et al. study provides two key messages: (a) the increased darunavir exposure can be attributed exclusively to SARS-CoV-2 and (b) this effect is switched off by the presence of concomitant HIV infection.

The mechanism by which HIV is able to mitigate the effects of SARS-CoV-2 on cytochromial enzyme activity and darunavir clearance cannot be derived from the present study given its retrospective naturalistic design. Despite these limitations, we believe that our case series, although small, may add another important piece to the puzzle well described by Cojutti et al. [1], showing that in patients with SARS-CoV-2 co-infected with HIV the presence of a pro-inflammatory state does not result in increased darunavir exposure. Even though the clinical relevance of these findings remains to be explored, this is a good example of how the co-existence of acute and chronic inflammatory conditions might provide unexpected and unpredictable results on drug disposition.

\section{Declarations}

Funding This study was carried out as part of our routine work.

Conflicts of interest/competing interests Cristina Gervasoni has received personal fees from MSD, ViiV, Gilead, and Janseen Cilag, outside the submitted work. Dario Cattaneo has received personal fees from MSD, ViiV, and Janseen Cilag, outside the submitted work. Mario Corbellino, Valeria Cozzi, and Marta Fusi have no conflicts of interest that are directly relevant to the content of this article.

Ethics approval The study was approved by our hospital's ethics committee (Comitato Etico Interaziendale Area 1, Milano, Italy).

Consent to participate Not applicable.

Consent for publication Not applicable.

Availability of data and material Not applicable.

Code availability Not applicable.

Authors' contributions Conception and study design: DC, CG; data acquisition: MC; data analysis: VC, MF. Each author contributed important intellectual content during manuscript drafting or revision, approved the submitted version of the manuscript, accepts personal accountability for the author's own contributions, and agrees to ensure that questions pertaining to the accuracy or integrity of any portion of the work are appropriately investigated and resolved.

\section{References}

1. Cojutti PG, Londero A, Della Siega P, Givone F, Fabris M, Biasizzo J, et al. Comparative population pharmacokinetics of darunavir in SARS-CoV-2 patients vs. HIV patients: the role of interleukin-6. Clin Pharmacokinet. 2020;59(10):1251-60. 
2. Gregoire M, Le Turnier P, Gaborit BJ, Veyrac G, Lecomte R, Boutoille D, et al. Lopinavir pharmacokinetics in COVID-19 patients. J Antimicrob Chemother. 2020;75(9):2702-4.

3. Schoergenhofer C, Jilma B, Stimpfl T, Karolyi M, Zoufaly A. Pharmacokinetics of lopinavir and ritonavir in patients hospitalized with Coronavirus Disease 2019 (COVID-19). Ann Intern Med. 2020;173(8):670-2.

4. Baldelli S, Corbellino M, Clementi E, Cattaneo D, Gervasoni C. Lopinavir/ritonavir in COVID-19 patients: maybe yes, but at what dose? J Antimicrob Chemother. 2020;75(9):2704-6.

5. Morgan ET. Impact of infectious and inflammatory disease on cytochrome P450-mediated drug metabolism and pharmacokinetics. Clin Pharmacol Ther. 2009;85(4):434-8.
6. Yu CY, Campbell SE, Sponseller CA, Small DS, Medlock MM, Morgan RE. Steady-state pharmacokinetics of darunavir/ritonavir and pitavastatin when co-administered to healthy adult volunteers. Clin Drug Investig. 2014;34(7):475-82.

7. Kakuda TN, Opsomer M, Timmers M, Iterbeke K, Van De Casteele T, Hillewaert V, et al. Pharmacokinetics of darunavir in fixed-dose combination with cobicistat compared with coadministration of darunavir and ritonavir as single agents in healthy volunteers. J Clin Pharmacol. 2014;54(8):949-57. 